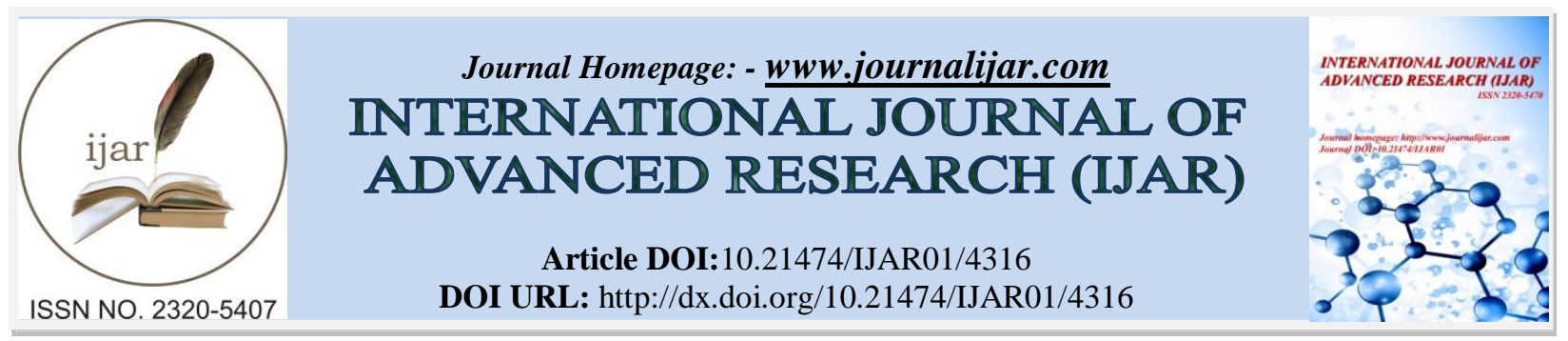

RESEARCH ARTICLE

\title{
ARTHRITE SEPTIQUE BILATERALE DES EPAULES SANS URETRITE A NEISSERIA GONORRHOEAEBILATERAL SHOULDER SEPTIC ARTHRITIS WITHOUT URETHRITIS CAUSED BY NEISSERIA GONORRHOEAE.
}

\begin{abstract}
Mohammed Sbiti, ${ }^{1, *}$, Jamal louaste ${ }^{2,3}$ and Lhoussaine louzi ${ }^{1,4}$.
1. Service de Bactériologie, Hôpital Militaire Moulay Ismail Meknès, Maroc.

2. Service de Traumatologie, Hôpital Militaire Moulay Ismail Meknès, Maroc.

3. Faculté de médecine et de pharmacie, université Mohammed Ben Abdellah - Fès, Maroc.

4. Faculté de médecine et de pharmacie, université Mohammed V-Souissi-Rabat, Maroc.
\end{abstract}

\section{Manuscript Info}

Manuscript History

Received: 21 March 2017

Final Accepted: 27 April 2017

Published: May 2017

\section{Key words:-}

Gonococcal arthritis, Biological diagnostics, Disseminated gonococcal infection, Risk factor

\section{Abstract}

Septic arthritis caused by Neisseria gonorrhoeae is mono or pauciarticular. They represent an uncommon cause of arthritis in sexually active adults. He is necessary to think of it even in the absence of urethritis.

We report the case of gonococcal arthritis without urethritis in an old man 48 years presented to the emergency department complaining of bilateral agreements shoulder pain in absence of risky sexual intercourse, associated with positive synovial fluid culture and negative blood cultures. Polyarthritis can occur, but a symmetrical involvement is unusual.

The diagnosis is above all microbiological; the samples are taken before any antibiotic therapy. Prompt recognition and treatment of this disease results in cure without aftereffects by used as initial therapy of a third-generation cephalosporins.

Copy Right, IJAR, 2017,. All rights reserved.

\section{Introduction:-}

L'infection gonococcique est encore répandue dans les pays en développement et principalement observée chez les jeunes adultes sexuellement actifs.

Les arthrites septiques à Neisseriagonorrhoeae (NG) sont rares, se développent chez des patients atteints d'infections disséminées à gonocoque (IDG).La prise en charge d'une arthrite septique est une urgence, il doit être le plus rapide possible afin d'éviter la destruction irréversible de l'articulation.

Le diagnostic repose sur un faisceau d'arguments cliniques bien décrit et doivent être confirmés rapidement par l'étude cytobactériologique de la ponction articulaire, qui reste l'examen diagnostique clef pour la mise en évidence de l'agent pathogène.

La rareté de cette infection associée à la difficulté d'isolement du gonocoque, nous incite à rapporter cette observation. 


\section{Présentation du cas:-}

Il s'agit d'un homme de 48 ans, s'est présenté au service des urgences pour douleurs à l'épaule bilatérale évoluant depuis deux semaines affectait sa capacité à effectuer ses activités, résistante aux AINS. Le patient n'était pas fébrile, Il n'a aucun antécédent médical ou chirurgical, ni de porte d'entrée cutanée, ni récente traumatisme autre que soulever des objets lourds au travail environ trois semaines avant. L'interrogatoire trouvait la notion brûlure urétrale et l'absence de rapport sexuel à risque déclaré.

L'examen clinique sur l'appareil locomoteur montrait, il y avait la douleur active et passive de mouvement dans les deux épaules. La douleur a été pire dans l'épaule droite et l'amplitude de mouvement était plus limitée sur la droite. Il n'y avait pas de chaleur ou de érythème soit épaule. Il n'y avait pas de douleur ou la limitation l'amplitude des mouvements du coude, du poignet ou la main sur soit le bras.

Il n'y a pas d'adénopathies satellite ni lésions cutanées. Les autres articulations sont sans anomalies. La radiographie de l'épaule droite a montré une acromio-claviculaire et les changements bénins hypertrophiquesdu rebord glénoïdien. Radiographies de l'épaule gauche ont montré la preuve d'un grand épanchement sans autre anomalie. Le patient a été opère et a subi des arthrotomieset les deux épaules étaient débridées et abondamment irrigué avec une solution saline normale.

Les examens biologiques ont objectivé un syndrome inflammatoire (VS à $45 \mathrm{~mm}$ à la première heure, CRP à 54 $\mathrm{mg} / \mathrm{ml}$ et fibrinogène $5,1 \mathrm{~g} / \mathrm{l}$ ) et un syndrome infectieux avec à l'hémogramme 12.800 leucocytes $/ \mathrm{mm} 3$ avec $84 \%$ de polynucléaires neutrophiles. La glycémie, urée et créatinine étaient normales.L'ECBU retrouvait moins de $10^{4}$ leucocytes et $210^{4}$ hématies par $\mathrm{ml}$ sans bactériurie. Le prélèvement urétral n'a isolé ni gonocoque ni chlamydia. Les sérologies VIH, VHB, VHC, chlamydia, syphilitique étaient négatives, de même que les hémocultures. Le bilan immunologique était normal avec un dosage de complément fraction $\mathrm{C} 4$ à $0,46 \mathrm{~g} / \mathrm{l}$ et des facteurs rhumatoïdes à $7,1 \mathrm{UI} / \mathrm{ml}$.

L'aspiration à l'aiguille de l'épaule droite a ramené $4,5 \mathrm{ml}$ de liquide épais, trouble. L'examen cytologique du liquide articulaire met en évidence $3.610^{6}$ leucocytes par mm3 dont la formule était $85 \%$ de polynucléaires neutrophiles et $15 \%$ de lymphocytes, avec absence de microcristaux.

L'examen microscopique après coloration de Gram ne met pas en évidence de germes. La culture (sur gélose au sang et gélose chocolat Polyvitex, incubée à $37^{\circ} \mathrm{C}$, en atmosphère contenant $5-10 \%$ de $\mathrm{CO} 2$ ) était positive après $48 \mathrm{~h}$, peu abondantes, faite de petites colonies, bombées, grisâtres avec oxydase et catalase positives. La coloration de Gram montre des cocci gram négatif en forme de grains de café, identifié comme Neisseriagonorrhoeae par les différentes caractéristiques biochimiques étudiées sur galeries Api NH.

L'antibiogramme objective une résistance aux fluoroquinolones avec une CMI $>0,06 \mathrm{mg} / 1$ pour la ciprofloxacine. La sensibilité est normale àla pénicilline $\mathrm{G}$, la tétracycline, la ceftriaxone, et la spectinomycine. La recherche de la production de bêta-lactamase par une technique chromogénique était négative.

Le patient mis sous Ceftriaxone IV pendant huit jours,et doxycycline par voie orale pour le traitement de N. gonorrhoeae et la chlamydia.

L'évolution clinique était favorable avec diminution des douleurs en quelques jours et suivi avec l'orthopédie et de physiothérapie.

\section{Discussion:-}

L'arthrite gonococcique peut résulter d'une dissémination bactérienne hématogène, notamment à NG, agent transmissible uniquement par voie sexuelle. En effet, 0,5 à $3 \%$ des infections muqueuses à gonocoque, le plus souvent asymptomatique, développent des infections disséminées multiviscérales [1]. La plus fréquente des atteintes au cours de cette dissémination est articulaire, les septicémies sont rares, peut causer une dermatite, exceptionnellement d'endocardite, de péricardite, de méningite [2]. Classiquement, la présentation clinique sous forme d'un syndrome arthrocutané fébrile : arthrites, ténosynovites (30 à $60 \%$ des cas) et une éruption érythématovésiculeuse (20 à $60 \%$ des cas) siégeant au niveau des membres.Les douleurs sont inflammatoires, vives, migratrices et l'épanchement articulaire est modéré. Les arthrites septiques gonococciques peuvent concerner toutes les articulations, elles se caractérisent par une atteinte polyarticulaire ou oligoarticulaire et asymétrique, sont localisées préférentiellement au niveau des genoux, poignets, chevilles etles doigts [1,3]. Les atteintes des hanches 
et sternoclaviculaires sont rares et se voient chez le patient VIH ou chez les utilisateurs de drogues injectables. Une arthritesymétrique localisée est plus rare, comme chez notre patient, souvent isolée et sans lésions cutanées ni ténosynovites.

Cette infection est principalement observée chez l'adulte jeune sexuellement actif avec une prédominance féminine.Les rapports sexuels non protégés avec partenaires multiples, la période menstruelle pour les femmes, l'infection à VIH, l'usage de drogue par voie intraveineuse et le bas niveau socio-économique sont des facteurs de risque de l'arthrite à gonocoque [3]. Chez l'homme, l'existence d'un portage asymptomatique, comme chez notre patient, semble constituer un facteur prédisposant à une dissémination hématogène. D'autres hypothèses comme un déficit complémentaire en fractions $\mathrm{C} 5$ à $\mathrm{C} 8$, d'un lupus ou bien une immunodépression ont été évoquées [4].

Neisseriagonorrhoeae est un germe à développement intracellulaire, pour produire une infection disséminée, NG échappe par divers moyens aux défenses de l'hôte [4]. Notamment, certaines protéines membranaires participent à la résistance à l'activité bactéricide du sérum. Sa virulence dépend de l'hôte (baisse de l'immunité, facteurs hormonaux), de son architecture structurale surtout sa composition membranaire (adhésines, lipo-oligosaccharide) et ses pili de typeIV [5].

Le diagnostic de certitude repose sur l'isolement du NG dans le liquide articulaire, le sang ou dans une porte d'entrée vénérienne, malgré l'absence de symptômes [1]. La ponction articulaire ramène un liquide trouble abondant contenant souvent entre 10.000 à 50.000 leucocytes $/ \mathrm{mm} 3$, sauf si le patient est immunosupprimé ou neutropénique. Le dosage du glucose sur le liquide articulaire démontre un taux effondré,les lactates sont habituellement augmentés et Le dosage de la CRP dans le liquide synovial semble également offrir des arguments en faveur de l'arthrite septique si la valeur est supérieure à $5 \mathrm{mg} / 1$ [6]. Des diplocoques intra- ou extraleucocytaires Gram négatifs sont retrouvés dans moins de $50 \%$ des cas. Le gonocoque étant un germe aérobie fragile avec ces multiples exigences métaboliques, sa mise en culture est difficile, elle doit être rapide après prélèvement nécessitant un milieu spécifique (gélose au sang cuit, Polyvitalex). L'inoculation directe de $1 \mathrm{ml}$ de liquide directement dans des flacons d'hémocultures peut améliorer le rendement diagnostique [7]. La culture du liquide articulaire est négative dans plus de $50 \%$ des arthrites purulentes, dans ce cas on a recours au test d'amplification des acides nucléiques (TAAN) pour recherche l'ADN bactérien par biologie moléculaire. Cette technique améliorée rapide plus sensibilité et plus spécificité que la culture. Cependant, le TAAN ne permet pas la réalisation d'un antibiogramme et donc la mise en évidence de résistances médicamenteuses [8].

L'étude de sensibilité aux antibiotiques est réalisée par la détermination des concentrations minimales inhibitrices (CMI) des différents antibiotiques recommandés (pénicilline G ou l'amoxicilline, la ceftriaxone, spectinomycine, tétracyclines, l'acide nalidixique, la ciprofloxacine ou l'ofloxacine, chloramphénicol et l'érythromycine).Les céphalosporines de troisième génération sont les antibiotiques de première intention. Néanmoins, une baisse de la sensibilité aux céphalosporines a commencé à apparaitre parmi les souches de NG au Japon, Hong Kong et en Corée de Sud [9].

La recherche de gonocoque au niveau du pharynx, de l'urètre et de l'anus est recommandée. Les hémocultures sont habituellement négatives en cas de monoarthrite et positives dans $30 \%$ des polyarthrites. Les examens systématiques concernent la mise en évidence de chlamydia ainsi que les sérologies syphilis, VIH et hépatite B.

L'hospitalisation est obligatoire pour confirmer le diagnosticdoit se faire aussi rapidement, s'assurer de l'absence de complications systématique (endocardite, méningite), et débuter une antibiothérapie par voie intraveineuse,après la réalisation des prélèvements bactériologiques [3].

Les céphalosporines de troisième générationinjectable sont le premier choix en monothérapie comme la ceftriaxone ( $1 \mathrm{~g} \mathrm{IM} / \mathrm{IV})$, le ceftizoxime ( $1 \mathrm{~g} \mathrm{IM} / \mathrm{IV}$ toutes les 8 heures) et le céfotaxime ( $1 \mathrm{~g} \mathrm{IV}$ toutes les 8 heures).En cas d'allergie aux $\beta$-lactamines ou chez une femme enceinte, il faut recourir à la spectinomycine (IM, $2 \mathrm{~g}$ en dose unique). La durée totale de traitement est très courte et de sept jours. Les antibiotiques parentéraux doivent être poursuivis jusqu'à ce que les symptômes se soient améliorés pendant 24 à 48 heures. La thérapie orale peut alors être prescrite pour compléter 7 jours d'antibiotique. Cefixime (400 mg deux fois / jour), ciprofloxacine (500 mg deux fois / jour)ou Levofloxacine (500 $\mathrm{mg}$ une fois / jour) [10].

L'arthrite gonococcique se caractérise par un excellent pronostic et une guérison se fait toujours sans séquelles lorsque le traitement approprié est rapidement initié. Le lavage avec du sérum physiologiquevise à décomprimer 
l'articulation et à évacuer les éléments inflammatoires susceptibles d'aggraver le pronostic articulaire.Le traitement chirurgical est exceptionnellement indiqué.

\section{Conclusion:-}

L'arthrite septique aiguë est une urgence médicale ayant une morbidité et mortalité considérables. Le diagnostic doit être le plus rapide possible afin d'éviter la destruction irréversible de l'articulation avec les conséquences qui en découlent sur la qualité de vie du patient.

L'antibiothérapie doit être rapidement mise en route après la réalisation des prélèvements bactériologiques. L'utilisation de ceftriaxone s'impose en première intention, elle est ensuite adaptée en fonction du résultat de l'antibiogramme.

\section{Références}

1. García-Arias M, Balsa A, Mola EM. Septic arthritis. Best Pract Res ClinRheumatol. 2011;25(3):407-21.

2. Guillot X, Delattre E, Prati C, Wendling D. Destructive septic arthritis of the sternoclavicular joint due to Neisseria gonorrhoeae. Joint Bone Spine. 2012;79 (5):519-20.

3. Bardin T. Gonococcal arthritis. Best Pract Res ClinRheumatol 2003;17:201-8.

4. DallaVestra M, Rettore C, Sartore P, et al. Acute septic arthritis: remember gonorrhea. RheumatolInt 2008; 29:81-5.

5. Ghosh SK, Zhao J, Philogene MC, Alzaharani A, Rane S, Banerjee A. Pathogenic consequences of Neisseria gonorrhoeae pilin glycan variation. Microbes Infect. 2004; 6 (7):693-701.

6. L Belkhir, T Schubert, N Devisscher, O Cornu, B Vandercam, JC Yombi. Arthrite septique : état de la question. Louvain Médical2008 ; 128(2):59-68.

7. John R. Papp, Julius Schachter, Charlotte A. Gaydos, Barbara Van Der Pol. Recommendations for the Laboratory-Based Detection of Chlamydia trachomatis and Neisseria gonorrhoeae-2014. MMWR. 2014; 63 (2):1-19.

8. Johan Chanal, François Lassau, Philippe Morand, Michel Janier, Nicolas Dupin. Actualités diagnostique et thérapeutique des infections à Neisseriagonorrhoeae. Presse Med. $2013 ; 42: 454-458$.

9. Barbee LA ,Dombrowski JC. Control of Neisseria gonorrhoeae in the era of evolving antimicrobial resistance. Infect Dis Clin North Am. 2013 Dec;27(4):723-37

10. Piszczek J, St Jean R, Khaliq Y. Gonorrhea: Treatment update for an increasingly resistant organism. Can Pharm J (Ott). 2015 Mar;148(2):82-9. 